\title{
ENFERMERIA BASADA EN LA EVIDENCIA: REVISIÓN DE ALCANCE
}

\author{
EVIDENCE-BASED NURSING: SCOPE REVIEW
}

\section{ENFERMAGEM BASEADA EM EVIDÊNCIAS: REVISÃO DO ESCOPO}

\author{
Amalia Silva Galleguillos* \\ Juan Vicente Beneit Montesinos** \\ Tamara Raquel Velasco Sanz*** \\ Esperanza Rayón Valpuesta****
}

\begin{abstract}
RESUMEN
Objetivos: Identificar la evidencia científica sobre el concepto, desarrollo y función, de la Enfermería Basada en la Evidencia (EBE) y analizar críticamente esta herramienta, sus elementos facilitadores y barreras para su implementación. Material y Método: Revisión de alcance según propuesta del Joanna Briggs Institute. Se consideró el rango de tiempo entre 1997 y 2019 para recuperar artículos y revisiones en inglés, portugués y español. Se utilizaron descriptores del tesauro Medical Subject Headings y términos booleanos para aplicar las estrategias de búsqueda en 4 bases de datos: PubMed, EBSCOhost, Scopus y Science Direct. Se complementó la búsqueda con la técnica de bola de nieve aplicada a la lista de referencias de los artículos relevantes seleccionados. Para evaluar la validez y el impacto de los resultados de los artículos de revisión y cualitativos se aplicó el Critical Appraisal Skills Programme Español (CASPE), y para estudios observacionales el Strengthening the Reporting of Observational studies in Epidemiology (STROBE). Para establecer los niveles de evidencia se utilizó Canadian Task Force on Preventive Health Care (CTPFCHC). Resultados: De 163.074 manuscritos iniciales, fueron seleccionados 44 para análisis y extraer datos referentes a: Historia de la EBE; Concepto y función de EBE; Etapas, barreras y facilitadores. Conclusiones: El concepto de EBE pone de manifiesto la necesidad de utilizar esta herramienta para planificar los cuidados mediante el uso del pensamiento crítico y reflexivo; la importancia de realizar el cuidado a la cabecera del paciente y cultivar un cuidado basado en la mejor evidencia, enmarcado, todo ello, en un contexto de humanización, así como considerar las barreras y facilitadores de esta herramienta.
\end{abstract}

Palabras clave: Enfermería Basada en la Evidencia; Revisión de alcance; Medicina Basada en la Evidencia; Historia; Barreras y facilitadores.

*Magíster en Enfermería Basada en la Evidencia e Investigación, Departamento Enfermería Universidad de Chile. Santiago, Chile. Doctoranda en Cuidados en Salud, Universidad Complutense de Madrid. Espańa. ORCID: https://orcid.org/0000-0003-23367262 Email: amaliasilva@uchile.cl. Autor de correspondencia.

**Médico, PhD en Medicina y Cirugía, Facultad de Enfermería, Fisioterapia y Podología, Universidad Complutense de Madrid, Madrid, España. ORCID: https://orcid.org/0000-0002-7510-4408 Email: jvbeneit@enf.ucm.es

***Enfermera, PhD en Cuidados en Salud, Hospital Universitario Clínico de San Carlos, Facultad de Enfermería, Fisioterapia y Podología. Universidad Complutense de Madrid, Madrid, Espańa. ORCID: https://orcid.org/0000-0002-6777-3337 Email: tavela01@ucm.es

****Enfermera, PhD en Antropología Social y Cultural, Facultad de Enfermería, Fisioterapia y Podología. Universidad Complutense de Madrid, Madrid, Espańa. ORCID: https:/orcid.org/0000-0003-0335-2362 Email: erayon@ucm.es 


\section{ABSTRACT}

Objective: To identify the scientific evidence on the concept, development and function of Evidence-Based Nursing (EBN) and to critically analyze this tool, its facilitating elements and barriers for implementation. Material and Method: Scoping review according to the proposal of the Joanna Briggs Institute. The period between 1997 and 2019 was considered to retrieve articles and reviews in English, Portuguese and Spanish. Medical Subject Headings thesaurus descriptors and Boolean operators were used to apply search strategies in 4 databases: Pubmed, EBSCOhost, Scopus, Science Direct. The search was complemented with the snowball technique applied to the reference list of the relevant selected articles. The Critical Appraisal Skills Programme Español (CASPE) was used to assess the validity and impact of the articles review and qualitative results, and the Strengthening the Reporting of Observational studies in Epidemiology (STROBE) was used for the observational studies. To establish the levels of evidence, The Canadian Task Force on Preventive Health Care (CTPFCHC) was used. Results: Out of 163,074 initial manuscripts, 44 were selected for analysis and data extraction regarding history of EBN; concept and function of EBN; stages, barriers and facilitators. Conclusions: The concept of EBN highlights the need to use this tool to plan nursing care through critical and reflective thinking; the importance of providing direct patient care and promoting care based on the best evidence, in a context of humanization and considering the tool's barriers and facilitators.

Key words: Evidence-Based Nursing; Scoping Review; Evidence-Based Medicine; History, Barriers and Facilitators.

\section{RESUMO}

Objetivo: Identificar as evidências científicas sobre o conceito, desenvolvimento e função da Enfermagem Baseada em Evidências (EBE) e analisar criticamente esta ferramenta, seus elementos facilitadores e as barreiras para sua implementação. Material e Método: Revisão do escopo de acordo com a proposta do Instituto Joanna Briggs. Considerou-se o período entre 1997 e 2019 para recuperar artigos e revisóes em inglês, português e español. Foram utilizados descritores do tesauro Medical Subject Headings e operadores booleanos para aplicar estratégias de pesquisa en 4 bancos de dados diferentes: Pubmed, EBSCOhost, Scopus, Science Direct. A pesquisa foi complementada com a técnica de bola de neve aplicada à lista de referência dos artigos selecionados relevantes. O Critical Appraisal Skills Programme Español (CASPE) foi usado para avaliar a validade e o impacto da revisão dos artigos e os resultados qualitativos, e para os estudos de observação utilizou-se o Strengthening the Reporting of Observational studies in Epidemiology (STROBE). A Canadian Task Force on Preventive Health Care (CTPFCHC) foi utilizada para establecer os níveis de evidência. Resultados: Dos 163.074 manuscritos iniciais, 44 foram selecionados para análise e extração de dados referentes à história da EBN; conceito e função da EBN; etapas, barreiras e facilitadores. Conclusóes: O conceito de EBN destaca a necessidade de utilizar esta ferramenta de planejamento de cuidado, através do uso do pensamento crítico e reflexivo; a importância de realizar o cuidado à beira do leito do paciente e promover cuidados baseados nas melhores evidências, em um contexto de humanização e considerando as barreiras e facilitadores desta ferramenta.

Palavras-chave: Enfermagem Baseada em Evidências; Revisão do Escopo; Medicina Baseada em Evidências; História, Barreiras e Facilitadores.

Fecha de recepción: 01/07/2021

Fecha de aceptación: 24/09/2021

\section{INTRODUCCIÓN}

Los profesionales de Enfermería tienen una responsabilidad con la ciencia y la sociedad. La capacidad de utilizar la ciencia para guiar la generación de conocimiento disciplinar y la gestión del cuidado, es un sello distintivo que debe ser parte de la práctica profesional. Para esto la investigación en enfermería es una herramienta valiosa que permite transformar la propia realidad en el contexto de los 
cuidados de la salud.

La Enfermería Basada en Evidencias (EBE), considerada como un nuevo paradigma de investigación, tiene sus orígenes en los años 70, deriva de la Medicina Basada en Evidencia (MBE) y en su evolución se le ha conocido como Práctica Basada en Evidencia (PBE), entre otros nombres, dependiendo de las conceptualizaciones otorgadas por diferentes autores ${ }^{(1)} \mathrm{y}$ en diferentes momentos. Este concepto, que nació hace más de 20 años en países como Reino Unido, Estados Unidos y Canadá ${ }^{(1)}$, ha recorrido un largo camino hasta la fecha. En la actualidad, la EBE es una herramienta declarada como un indicador de calidad y los beneficios que otorga su utilización movilizan a muchos actores ${ }^{(1)}$ : se reduce la brecha entre la teoría y la práctica en la aplicación de los cuidados, que beneficia tanto a las personas, como a las instituciones y los países; acorta el tiempo hospitalario que se traduce en un retorno más temprano a la vida familiar y en una mejor calidad de vida para los pacientes. De esta forma, la institución, indirectamente, se beneficia porque disminuye el gasto sanitario y la persona tiene una pronta integración a la actividad laboral, que, a su vez, contribuye también a una mayor producción a nivel país.

La utilización de la investigación por parte de enfermería se observa claramente cuando la EBE sustenta la práctica clínica y surge la necesidad de revisar en profundidad la temática para conocer su evolución, actualizar sus conceptos e identificar las estrategias que permiten avanzar en la utilización de esta herramienta, sobre todo en aquellos países de Latinoamérica que aún no han integrado este cambio en la forma de entregar cuidados de salud.

Por lo anterior, el sustento teórico metodológico elegido para este estudio fue lo señalado por el Joanna Briggs Institute $(J B I)^{(2)}$ respecto a las scoping review o revisiones de alcance: se realizan como una etapa previa a una revisión sistemática para mapear los conceptos claves que sustentan un área de investigación, así como para aclarar definiciones de trabajo y/o los límites conceptuales de un tema, examinar y aclarar áreas más amplias con el fin de identificar brechas en la evidencia, aclarar conceptos clave e informar sobre los tipos de evidencia y la práctica en un área temática. "El valor de las revisiones de alcance, para la práctica basada en la evidencia, radica en el examen de un área más amplia para identificar brechas en la base de conocimiento de investigación"(2).

Con todo, los objetivos de esta revisión fueron: 1) Identificar la evidencia científica para conocer el concepto, historia, desarrollo y función de la EBE y 2) Analizar críticamente esta herramienta, sus elementos facilitadores y barreras para su implementación.

\section{MATERIAL Y MÉTODO}

1. Diseño: Planteada la siguiente pregunta: ¿Cómo ha sido el desarrollo de la EBE, sus definiciones, nuevos conceptos, funciones, elementos facilitadores y barreras para su implementación?, se desarrolló una revisión de alcance, y con el fin de cautelar el rigor metodológico se sistematizó el proceso siguiendo los pasos propuestos en el Manual de revisión del $J B I$, apoyado en el marco de revisión de alcance propuesto por Peter et al. en el año $2015^{(3)}$

2. Estrategia de búsqueda: Se efectuó la búsqueda en las bases de datos PubMed, EBSCOhost, Scopus y Science Direct, aplicando una estrategia de búsqueda diseñada con descriptores del tesauro Medical Subject Heading (MeSH), que se relacionaran con los objetivos de la revisión, vinculados con operadores booleanos (Tabla 1).

Además, se utilizó una búsqueda manual en la lista de referencias de los artículos seleccionados con la técnica de bola de nieve, de acuerdo con el nuevo aporte que estos artículos presentaban.

3. Criterios de inclusión y exclusión: Se consideraron todos aquellos artículos científicos que abarcaran EBE, su origen, etapas del proceso, barreras y elementos facilitadores para su aplicación. Fueron excluidos artículos en donde EBE se aplicaba en forma específica para la prestación de algún determinado cuidado. Se consideró el rango de tiempo entre el año 1995, dado que los primeros escritos de EBE se remontan a esta fecha ${ }^{(4)}$ y el año 2019, determinado por la búsqueda manual realizada. Se incluyeron artículos en inglés, español y portugués (Tabla 1).

4. Extracción de datos: Como resultado de la primera búsqueda, se obtuvo un total de 163.074 artículos, de los cuales fueron seleccionados 18 para resolver el primer objetivo, según se detalla en 
la Figura 1. Para responder al segundo objetivo, en la Figura 2 se detalla la derivación de pasos hasta llegar a seleccionar 26 artículos que presentaban la información desde los diferentes paradigmas investigativos.

Para evaluar la validez interna (rigurosidad metodológica), validez externa (extrapolación de los datos) y el impacto (fracción de exposición en los casos, diferencia de riesgo, entre otras) de los resultados de los artículos seleccionados, se utilizaron las siguientes herramientas: Critical Appraisal Skills Programme en Español $(C A S P E)^{(5)}$ para las revisiones sistemáticas y los estudios cualitativos, Strengthening the Reporting of Observational studies in Epidemiology (STROBE) para los estudios observacionales y Canadian Task Force on Preventive Health Care (CTPFCHC) para medir los niveles de evidencia de los artículos.

\section{RESULTADOS}

Caracterización de los artículos: La Tabla 1 informa la matriz que caracteriza a los 44 trabajos seleccionados y a los objetivos que respondían. Los 18 artículos seleccionados para el primer objetivo fueron principalmente revisiones narrativas. Los diseños de investigación de los 26 estudios incorporados para el segundo objetivo fueron mayoritariamente cuantitativos (16), de investigaciones primarias, con una población de estudio del ámbito hospitalario y de diferentes países americanos, europeos, asiáticos y africanos. Todos los artículos fueron calificados con un nivel de evidencia III.

Tabla 1. Objetivos, bases de datos y estrategias de búsqueda empleada en la cadena de recuperación bibliográfica.

\begin{tabular}{|c|c|c|c|}
\hline Objetivos & Bases de datos & Cadena de búsqueda & $\begin{array}{c}\text { Registros } \\
\text { recuperados }\end{array}$ \\
\hline \multirow{8}{*}{$\begin{array}{c}\text { Identificar la } \\
\text { evidencia científica } \\
\text { para conocer el } \\
\text { concepto, historia, } \\
\text { desarrollo y función } \\
\text { de la EBE }\end{array}$} & PubMed & 1. Evidence-Based Medicine or Evidence-based Nursing & 26.497 \\
\hline & \multirow{3}{*}{ Cinhal } & 2. (Evidence-Based Nursing) [Mesh] and (History) [Mesh] & 141 \\
\hline & & 1. Evidence-Based Medicine or Evidence-based Nursing & 4.543 \\
\hline & & 2. Evidence-Based Nursing and History & 24 \\
\hline & \multirow[t]{2}{*}{ Scopus } & 1. Evidence-Based Medicine or Evidence-based Nursing & 17.696 \\
\hline & & 2. Evidence-Based Nursing and History & 398 \\
\hline & \multirow[t]{2}{*}{ Science Direct } & 1. Evidence-Based Medicine or Evidence-based Nursing & 114.338 \\
\hline & & 2. Evidence-Based Nursing and History & 624 \\
\hline \multirow{8}{*}{$\begin{array}{c}\text { Analizar } \\
\text { críticamente esta } \\
\text { herramienta, } \\
\text { sus elementos } \\
\text { facilitadores y } \\
\text { barreras para su } \\
\text { implementación. }\end{array}$} & PubMed & 1. (Evidence-Based Nursing) and barriers. & 1.814 \\
\hline & \multirow{3}{*}{ Cinhal } & $\begin{array}{l}\text { 2. (Evidence-Based Nursing) [Mesh] and (barriers) [Mesh] } \\
\text { and (implementation ) [Mesh] }\end{array}$ & 649 \\
\hline & & 1. Evidence-Based Nursing and barriers. & 930 \\
\hline & & 2. Evidence-Based Nursing and barriers and implementation & 334 \\
\hline & \multirow[t]{2}{*}{ Scopus } & 1. Evidence-Based Nursing and barriers. & 1.489 \\
\hline & & 2. Evidence-Based Nursing and barriers and implementation & 868 \\
\hline & \multirow[t]{2}{*}{ Science Direct } & 1. Evidence-Based Nursing and barriers & 2.963 \\
\hline & & 2. Evidence-Based Nursing and barriers and implementation & 871 \\
\hline
\end{tabular}




\begin{tabular}{|c|c|c|c|c|c|c|}
\hline Pasos & & Pubmed & Cinhal & Scopus & Science Direct & $\begin{array}{c}\text { Búsqueda } \\
\text { manual }\end{array}$ \\
\hline \multicolumn{2}{|c|}{\begin{tabular}{|l} 
I. Busqueda: Evidence-based Medicine OR Evidence-Based Nursing \\
(Total= 163.074 )
\end{tabular}} & 26.497 & 4.543 & 17.696 & 114.338 & 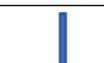 \\
\hline Excluidos $=161.896$ & & & & & & \\
\hline \multicolumn{2}{|c|}{$\begin{array}{l}\text { II. Selección: artículos sobre Evidence- Based Nursing AND History } \\
\text { (Total=1.178) }\end{array}$} & 141 & 24 & 389 & 624 & \\
\hline Excluidos $=1.165$ & & & & & & \\
\hline \multicolumn{2}{|c|}{$\begin{array}{c}\text { III Paso. Selección según criterios de inclusión y exclusión } \\
\text { (Total=13) }\end{array}$} & 5 & 6 & 1 & 1 & \\
\hline \multicolumn{4}{|c|}{ IV Paso. Búsqueda utilizando la técnica de bola de nieve } & & & Total $=5$ \\
\hline
\end{tabular}

Figura 1. Selección para primer objetivo: tema Historia.

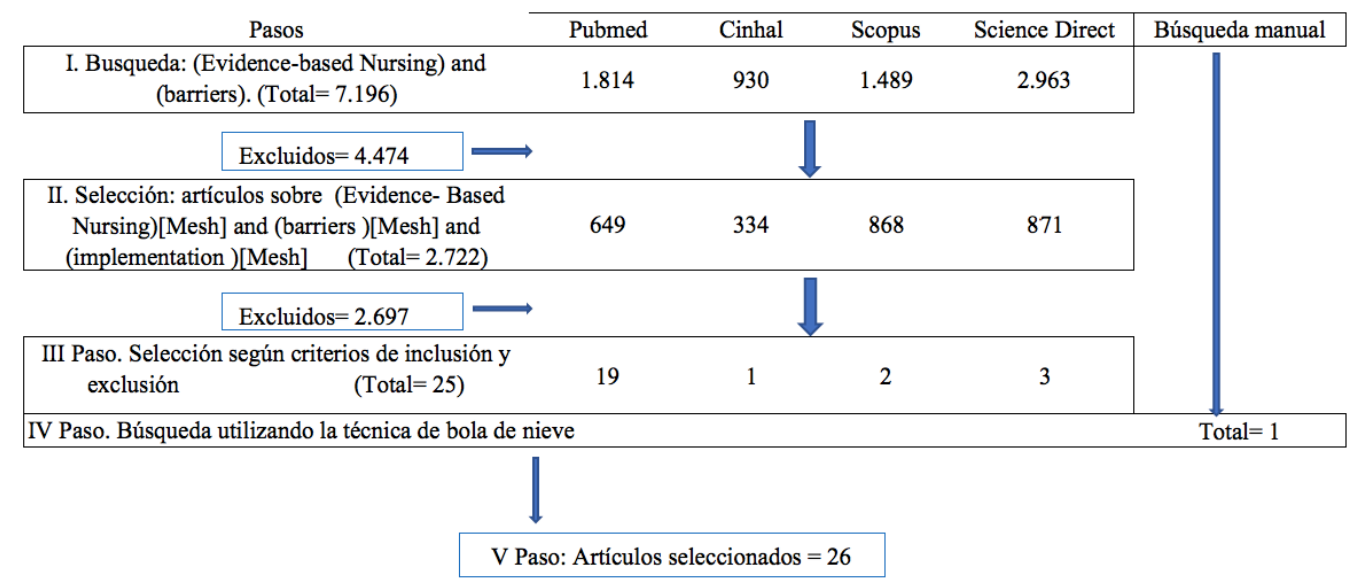

Figura 2. Selección para segundo objetivo: tema Barreras y Facilitadores.

Origen, concepto, funciones y etapas de la EBE: En relación a los orígenes, varios artículos ${ }^{(1,6-15)}$ mencionan a Archibald Cochrane, epidemiólogo del Reino Unido, como uno de los precursores de este movimiento. En su libro, publicado en 1972 y titulado "Eficiencia y Efectividad de los sistemas sanitarios" ${ }^{\prime(7)}$, realiza una fuerte crítica al estamento médico por no utilizar la investigación para resolver los problemas de salud de la población. Su postulado era usar, principalmente, los ensayos clínicos controlados y randomizados para la toma de decisiones clínicas, porque proporcionaban la forma más confiable de evidencia.

Otros autores ${ }^{(7)}$ mencionan que en 1980 aparecieron las primeras publicaciones orientadas a revisar críticamente estudios publicados en revistas médicas. Un grupo de epidemiólogos de la Facultad de Ciencias de la Salud de la Universidad de Mc Master publicaron en los 90 una descripción del análisis correcto de la evidencia científica, dando origen a lo que se llamaría, posteriormente, Medicina Basada en Evidencia (MBE).

Gracias al trabajo de Cochrane, su influencia y la necesidad de actualizar las revisiones sistemáticas de los ensayos clínicos aleatorizados, se fundó el Cochrane Center en Oxford, Inglaterra, en 1992 y un año más tarde la Cochrane Collaboration, cuyo principal objetivo es seguir sus postulados para implementar intervenciones sanitarias informadas con base en la mejor y más actualizada evidencia. 
Tabla 2. Características de los artículos seleccionados, período 1997-2019 (n=44).

\begin{tabular}{|c|c|c|c|c|}
\hline (cita) Ańo, País & $\begin{array}{l}\text { Bases de datos/ } \\
\text { Búsqueda } \\
\text { manual }\end{array}$ & Palabras clave & Tipo de estudio & Variable \\
\hline (1)2013, EE.UU. & PUBMED & $\begin{array}{l}\text { Evidence-based nursing, evidence- } \\
\text { based practice, research utilization }\end{array}$ & Revisión Narrativa & Historia \\
\hline (6) 2006, Venezuela & PUBMED & Medicina-basada en evidencia. & $\begin{array}{l}\text { Revisión de Ensayo } \\
\text { Clínico }\end{array}$ & Historia \\
\hline (7)2017, Canadá & Scopus & $\begin{array}{l}\text { Evidence-based nursing; Nursing } \\
\text { history. }\end{array}$ & Artículo Original & Historia \\
\hline${ }^{(8)}$ 2007, México & PUBMED & Medicina basada en evidencias. & Revisión Narrativa & Historia \\
\hline (9) 2009, Reino Unido & PUBMED & Evidence-Based Nursing & Revisión Crítica & $\begin{array}{l}\text { Historia- } \\
\text { Conceptos }\end{array}$ \\
\hline${ }^{(10)} 2017$, EE.UU. & CINAHL & $\begin{array}{l}\text { evidence-based practice, application } \\
\text { to practice, history }\end{array}$ & $\begin{array}{l}\text { Revisión y estudio } \\
\text { de caso }\end{array}$ & $\begin{array}{l}\text { Historia- } \\
\text { Conceptos }\end{array}$ \\
\hline (11)2000, EE.UU. & CINAHL & & Revisión Narrativa & Historia \\
\hline${ }^{(12)} 2004$, EE.UU. & CINAHL & & Revisión Narrativa & Historia \\
\hline (13)2011, España & CINAHL & & Revisión Narrativa & Historia \\
\hline (14)2011, Costa Rica & Búsqueda manual & $\begin{array}{l}\text { Evidencia, enfermería, investigación } \\
\text { en enfermería, práctica de } \\
\text { enfermería. }\end{array}$ & $\begin{array}{l}\text { Revisión- } \\
\text { Reflexión }\end{array}$ & Historia \\
\hline${ }^{(15)} 2001$, Canadá & CINAHL & & Revisión Narrativa & Historia \\
\hline (16) 2010, Chile & PUBMED & $\begin{array}{l}\text { Investigación en Enfermería; } \\
\text { práctica basada en evidencia. }\end{array}$ & Revisión Narrativa & $\begin{array}{l}\text { Historia- } \\
\text { Conceptos }\end{array}$ \\
\hline${ }^{(17)} 2004$, Canadá & PUBMED & $\begin{array}{l}\text { Investigación en Enfermería; } \\
\text { práctica basada en evidencia. }\end{array}$ & Reflexión & Historia \\
\hline (18)2003, Chile & PUBMED & $\begin{array}{l}\text { Clinical Medicine; Decission } \\
\text { Support Systems, Clinical; Evidence } \\
\text { based Medicine; Literature, modern }\end{array}$ & Revisión Narrativa & $\begin{array}{l}\text { Historia- } \\
\text { Conceptos } \\
\text { Etapas }\end{array}$ \\
\hline${ }^{(19)} 2011$, España & CINHAL & & Revisión Narrativa & $\begin{array}{l}\text { Historia- } \\
\text { Conceptos } \\
\text { Etapas }\end{array}$ \\
\hline${ }^{(20)} 1999$, España & $\begin{array}{l}\text { Búsqueda } \\
\text { manual }\end{array}$ & & Revisión Narrativa & $\begin{array}{l}\text { Historia- } \\
\text { Conceptos } \\
\text { Etapas }\end{array}$ \\
\hline (21)2001, Reino Unido & $\begin{array}{l}\text { Búsqueda } \\
\text { manual }\end{array}$ & & Revisión Narrativa & $\begin{array}{l}\text { Historia- } \\
\text { Conceptos } \\
\text { Etapas }\end{array}$ \\
\hline (22) 2007, Chile & $\begin{array}{l}\text { Búsqueda } \\
\text { manual }\end{array}$ & $\begin{array}{l}\text { Evidence based nursing, nursing } \\
\text { research, barriers and strategies for } \\
\text { implementation }\end{array}$ & Revisión Narrativa & $\begin{array}{l}\text { Historia- } \\
\text { Barreras }\end{array}$ \\
\hline $\begin{array}{l}{ }^{(23)} 2000 \text {, Irlanda del } \\
\text { Norte }\end{array}$ & PUBMED & & $\begin{array}{l}\text { Cuantitativo- } \\
\text { Descriptivo }\end{array}$ & $\begin{array}{l}\text { Barreras- } \\
\text { Facilitadores }\end{array}$ \\
\hline (24) 2003 , España & Science Direct & $\begin{array}{l}\text { Enfermería basada en la evidencia. } \\
\text { Investigación. Barreras. Facilitadores. }\end{array}$ & $\begin{array}{l}\text { Cuantitativo- } \\
\text { Descriptivo }\end{array}$ & $\begin{array}{l}\text { Barreras- } \\
\text { Facilitadores }\end{array}$ \\
\hline
\end{tabular}


Continuación Tabla 2.

\begin{tabular}{|c|c|c|c|c|}
\hline (cita) Año, País & $\begin{array}{l}\text { Bases de datos/ } \\
\text { Búsqueda } \\
\text { manual }\end{array}$ & Palabras clave & Tipo de estudio & Variable \\
\hline (25)2010, España & Science Direct & & $\begin{array}{l}\text { Cuantitativo- } \\
\text { Descriptivo }\end{array}$ & $\begin{array}{l}\text { Barreras- } \\
\text { Facilitadores }\end{array}$ \\
\hline (26)2019, Australia & PUBMED & $\begin{array}{l}\text { Health service evaluation; } \\
\text { implementation science; evidence- } \\
\text { based practice; best practice } \\
\text { guidelines; program evaluation; } \\
\text { nursing and midwifery }\end{array}$ & Estudio Mixto & $\begin{array}{c}\text { Barreras- } \\
\text { Facilitadores }\end{array}$ \\
\hline (27)2012, EE.UU. & PUBMED & & $\begin{array}{l}\text { Estudio } \\
\text { Descriptivo }\end{array}$ & $\begin{array}{l}\text { Barreras- } \\
\text { Facilitadores }\end{array}$ \\
\hline${ }^{(28)}$ 2019, Reino Unido & PUBMED & $\begin{array}{l}\text { community nursing; community/ } \\
\text { home care; evidence-based practice; } \\
\text { implementation; systematic review }\end{array}$ & $\begin{array}{c}\text { Revisión } \\
\text { sistemática mixta }\end{array}$ & $\begin{array}{c}\text { Barreras- } \\
\text { Facilitadores }\end{array}$ \\
\hline (29) 2019 , China & Scopus & $\begin{array}{l}\text { community nurse, evidence-based } \\
\text { practice, literature review, nurses, } \\
\text { systematic review }\end{array}$ & Revisión Narrativa & $\begin{array}{l}\text { Barreras- } \\
\text { Facilitadores }\end{array}$ \\
\hline (30)2008, EE.UU. & PUBMED & & $\begin{array}{l}\text { Cuantitativo- } \\
\text { Descriptivo }\end{array}$ & $\begin{array}{l}\text { Barreras- } \\
\text { Facilitadores }\end{array}$ \\
\hline (31)2019, Corea del Sur & PUBMED & & $\begin{array}{l}\text { Cuantitativo- } \\
\text { Descriptivo }\end{array}$ & Barreras \\
\hline (32)2019, Arabia Saudí & PUBMED & $\begin{array}{l}\text { Saudi Arabia; attitudes; evidence- } \\
\text { based practice; implementation; } \\
\text { knowledge; nurses }\end{array}$ & $\begin{array}{l}\text { Cuantitativo- } \\
\text { Correlacional }\end{array}$ & Barreras \\
\hline (33)2016, Corea del Sur & PUBMED & $\begin{array}{l}\text { Curriculum; Evidence based } \\
\text { practice; Nursing faculties; Self- } \\
\text { efficacy }\end{array}$ & Estudio Mixto & $\begin{array}{c}\text { Barreras- } \\
\text { Facilitadores }\end{array}$ \\
\hline (34) 2018, Brasil & CINAHL & $\begin{array}{l}\text { enfermería basada en evidencias; } \\
\text { práctica clínica basada en evidencias; } \\
\text { hospitales; Enfermeras Clínicas; } \\
\text { Investigación en Enfermería. }\end{array}$ & $\begin{array}{l}\text { Revisión } \\
\text { Integrativa }\end{array}$ & $\begin{array}{l}\text { Barreras- } \\
\text { Facilitadores }\end{array}$ \\
\hline (35) 2014 , Oman & PUBMED & & $\begin{array}{l}\text { Cuantitativo- } \\
\text { Descriptivo }\end{array}$ & $\begin{array}{l}\text { Barreras- } \\
\text { Facilitadores }\end{array}$ \\
\hline (36) 2013, España & $\begin{array}{c}\text { Búsqueda } \\
\text { manual }\end{array}$ & $\begin{array}{l}\text { práctica clínica basada en evidencias; } \\
\text { obstáculos. }\end{array}$ & $\begin{array}{l}\text { Original breve / } \\
\text { Cualitativo }\end{array}$ & Barreras \\
\hline (37)2019, Irán & Scopus & $\begin{array}{l}\text { barriers; evidence-based } \\
\text { management; evidence-based } \\
\text { nursing; facilitators; nursing } \\
\text { managers' perspective }\end{array}$ & $\begin{array}{l}\text { Cuantitativo- } \\
\text { Descriptivo }\end{array}$ & $\begin{array}{l}\text { Barreras- } \\
\text { Facilitadores }\end{array}$ \\
\hline (38)2018, Canadá & PUBMED & $\begin{array}{l}\text { qualitative study, evidence-based } \\
\text { practice, Barriers, Facilitators, China }\end{array}$ & $\begin{array}{l}\text { Cualitativa- } \\
\text { Descriptiva }\end{array}$ & $\begin{array}{l}\text { Barreras- } \\
\text { Facilitadores }\end{array}$ \\
\hline $\begin{array}{l}\text { (39) } 2018 \text {, Trinidad y } \\
\text { Tobago }\end{array}$ & PUBMED & $\begin{array}{l}\text { Bahamas; barriers; evidence-based } \\
\text { practice; facilitators; nursing }\end{array}$ & $\begin{array}{l}\text { Cuantitativo- } \\
\text { Descriptivo }\end{array}$ & $\begin{array}{l}\text { Barreras- } \\
\text { Facilitadores }\end{array}$ \\
\hline $\begin{array}{l}{ }^{(40)} \text { 2011, Holanda, } \\
\text { Países Bajos }\end{array}$ & PUBMED & & $\begin{array}{l}\text { Cuantitativo- } \\
\text { Descriptivo }\end{array}$ & Barreras \\
\hline
\end{tabular}


Continuación Tabla 2.

\begin{tabular}{|c|c|c|c|c|}
\hline (cita) Año, País & $\begin{array}{c}\text { Bases de datos/ } \\
\text { Búsqueda } \\
\text { manual }\end{array}$ & Palabras clave & Tipo de estudio & Variable \\
\hline (41) 2007, Irán & PUBMED & & $\begin{array}{l}\text { Cualitativa- } \\
\text { Descriptiva }\end{array}$ & $\begin{array}{l}\text { Barreras- } \\
\text { Facilitadores }\end{array}$ \\
\hline (42) 2005, Reino Unido & PUBMED & & $\begin{array}{l}\text { Estudio de caso y } \\
\text { método mixto }\end{array}$ & $\begin{array}{l}\text { Barreras- } \\
\text { Facilitadores }\end{array}$ \\
\hline${ }^{(43)} 2011$, Singapore & PUBMED & & $\begin{array}{l}\text { Cuantitativo- } \\
\text { Descriptivo }\end{array}$ & $\begin{array}{c}\text { Barreras- } \\
\text { Facilitadores }\end{array}$ \\
\hline (44) 2014, Irán & PUBMED & & $\begin{array}{l}\text { Cuantitativo- } \\
\text { Descriptivo }\end{array}$ & $\begin{array}{c}\text { Barreras- } \\
\text { Facilitadores }\end{array}$ \\
\hline (45) 2019 , Oman & PUBMED & $\begin{array}{l}\text { attitudes; competence; cultural } \\
\text { implications; evidence-based } \\
\text { practice; knowledge; multicountry } \\
\text { research; nursing student; skills }\end{array}$ & $\begin{array}{l}\text { Cuantitativo- } \\
\text { Descriptivo }\end{array}$ & $\begin{array}{c}\text { Barreras- } \\
\text { Facilitadores }\end{array}$ \\
\hline (46)2009, EE.UU. & PUBMED & & $\begin{array}{l}\text { Cuantitativo- } \\
\text { Descriptivo }\end{array}$ & $\begin{array}{c}\text { Barreras- } \\
\text { Facilitadores }\end{array}$ \\
\hline (47)2014, Colombia & PUBMED & & $\begin{array}{l}\text { Cualitativa- } \\
\text { Descriptiva }\end{array}$ & $\begin{array}{c}\text { Barreras- } \\
\text { Facilitadores }\end{array}$ \\
\hline${ }^{(48)} 2015$, Corea del Sur & PUBMED & $\begin{array}{l}\text { critical thinking disposition; } \\
\text { evidence-based practice; nursing; } \\
\text { research barriers; structure equation } \\
\text { modeling }\end{array}$ & $\begin{array}{l}\text { Cuantitativo- } \\
\text { Descriptivo }\end{array}$ & $\begin{array}{c}\text { Barreras- } \\
\text { Facilitadores }\end{array}$ \\
\hline
\end{tabular}

En 1990, Gordon Guyatt acuñó el término en un documento informal destinado a los estudiantes residentes de Medicina Interna de la Universidad de McMaster, en Canadá(1, 7,8). En este artículo Guyatt plantea un nuevo paradigma en medicina, basado en la ciencia de la investigación clínica, superando el modelo tradicional que se apoyaba en el conocimiento empírico ${ }^{(8)}$.

En 1991 Guyatt utilizó por primera vez el término de $\mathrm{MBE}$ y rápidamente se extendió a otras profesiones de la salud ${ }^{(8)}$. Cinco años más tarde, en 1996, Sackett define MBE al señalar que los profesionales de la salud deberían aplicar "el uso concienzudo, explícito y juicioso de la mejor evidencia para tomar decisiones sobre el cuidado de pacientes individuales"(1, 7-10, 13, 14). Pasados cuatro años, nuevamente Sackett ${ }^{(7,10)}$ modifica la definición para incluir a profesionales y pacientes en esta conceptualización, que nuevamente enfatizaba la necesidad de integrar la mejor evidencia científica procedente de la investigación sistemática, junto a la habilidad y buen juicio obtenido de la experiencia clínica, considerando, además, las preferencias y valores del paciente.
Junto con el concepto de $\mathrm{MBE}$ se puede encontrar el concepto de Práctica Basada en la Evidencia (PBE), utilizada por otras profesiones de salud, tales como fisioterapia, audiología, odontología y trabajo social, entre otras, que la han adoptado como propia $^{(10)}$.

Una de las diferencias desde sus inicios entre EBE y MBE, dado el ámbito de desempeño de enfermería, es que su enfoque teórico no sólo se resuelve desde el alcance experimental de los estudios, sino que la investigación de tipo cualitativa también debe ser incorporada. Autores como Popay plantean que ambos tipos de investigación son complementarios para enfermería ${ }^{(13)}$.

En cuanto a la Historia de la Enfermería Basada en la Evidencia, se menciona a Florence Nightingale como una de las precursoras de esta práctica ${ }^{(7,10)}$, tanto por su experiencia en la guerra de Crimea, como en el hospital en Scutari de Turquía. Si bien es cierto que los términos PBE o EBE, en el tiempo de Nightingale, no existían, su aporte fue señalar que con el uso de la experimentación y una mirada crítica al cuidado, era posible influir para lograr resultados positivos en los pacientes. Florence 
Nightingale fue una estadística apasionada, y en muchos de sus escritos se observa que usó la evidencia $^{(7,10,15)}$ y la recopilación sistemática de datos para explorar temas como la mortalidad, diferenciada entre subgrupos de población, la atención brindada por enfermeras capacitadas o no capacitadas, el exceso de mortalidad después del parto, o el uso de la evidencia para guiar decisiones políticas. Una de sus preocupaciones era compartir estos conocimientos y por ello intentó promover una cátedra denominada "Física Social", destinada a quienes asistían a formarse en la Universidad de Oxford, la mayoría funcionarios públicos y políticos, pero se rechazó su propuesta porque no fue entendida por las autoridades de la época ${ }^{(15)}$.

Enfermería también se ha involucrado en esta forma de cuidado basada en la investigación y recibe el nombre de Enfermería Basada en la Evidencia (EBE) en 1997(11, 14). Países como Canadá, Estados Unidos y Reino Unido fueron los primeros en adoptar esta forma de efectuar la práctica clínica del cuidado científico ${ }^{(14,16)}$. Sus primeros indicios se atribuyen a la década de 1960, en donde se evalúo la efectividad clínica, y se sugirió que los tratamientos efectivos se usaban incorrectamente, mientras que los tratamientos ineficaces a menudo se usaban con frecuencia $^{(16)}$. Los orígenes de la EBE, en el ámbito académico, parten en la Universidad de York, Reino Unido ${ }^{(13)}$, cuando se creó el primer centro de EBE, cuyo objetivo fue unir la investigación y las necesidades de la práctica. Desde Canadá surge la primera publicación relacionada con la investigación en Enfermería, en 1972, de la canadiense Helen Shore.

De igual forma, se afirma que en América del Norte, la División de Enfermería de los Estados Unidos proporcionó recursos económicos para financiar grandes proyectos para la utilización de la investigación, como parte del Proyecto de la Comisión interestatal occidental para la educación superior en Enfermería WICHEN, sobre el desarrollo de la investigación en Enfermería ${ }^{(17)}$. A partir de ahí, el uso de la investigación en enfermería aumentó y las enfermeras investigadoras se dieron a la tarea de crear modelos para la utilización de esta investigación en la práctica. Entre otros, algunos ejemplos son el Modelo de Investigación en Práctica de Iowa, publicado en 1994, o los Modelos de Investigación colaborativa, publicados en 1995. Este movimiento de EBE también motivó la creación de centros encargados de la síntesis de este nuevo conocimiento derivado de las investigaciones de enfermería. Es así como en 1996, en Australia y Nueva Zelanda, surge el Joanna Briggs Institute y centros colaboradores; en Reino Unido The Center for Evidence-Based Nursing at the University of York. En 1997 se celebraron en Inglaterra las primeras conferencias sobre $\mathrm{EBE}^{(13,14)}$. En Canadá también se establecieron importantes agencias nacionales de financiamiento de la salud, como en 1997 la Fundación Canadiense de Investigación de Servicios de Salud (CHSRF) o los Institutos Canadienses de Investigación en Salud (CIHR), en 2000.

En 1998, en Inglaterra se inicia la divulgación de estas investigaciones por medio de la revista Evidence-Based Nursing y ese mismo año, en los Estados Unidos, se crea The Sarah Cole Hirsch Institute ${ }^{(16)}$.

Respecto al concepto de PBE en enfermería, se define como "un enfoque de resolución de problemas para la toma de decisiones clínicas y evaluación, y valores de preferencia de los pacientes dentro de un contexto de la atención” y se afirma que se basa en la toma de decisiones y se utiliza para optimizar los resultados de los pacientes, mejorar la práctica clínica y garantizar la responsabilidad en enfermería $^{(7)}$.

Respecto al concepto y función de la EBE, la literatura señala diversas definiciones y utilidades surgidas desde organismos internacionales de enfermería, eventos del colectivo sobre la materia o investigadores, a saber: "uso concienzudo, explícito y uso juicioso de la información basada en la investigación derivada de la teoría para tomar decisiones sobre la prestación de atención a individuos o grupos de pacientes que reflejan las necesidades y preferencias del paciente"(1, 9); "uso consciente y explícito, desde el mundo del pensamiento de las enfermeras, de las ventajas que ofrece el modelo positivista de síntesis de la literatura científica de la medicina basada en la evidencia, integrado en una perspectiva crítica, reflexiva y fenomenológica tal que haga visibles perspectivas de la salud invisibilizadas por el pensamiento hegemónico"(13); "concepto amplio que tiene como objeto mejorar la seguridad del paciente, reducir los costos de atención médica y, en última instancia, proporcionar un marco que apoye la toma de decisiones en situaciones específicas del paciente ${ }^{(6)}$; 
"el cuidado concerniente a la incorporación de la evidencia de la investigación, expertez clínica y la preferencia de los pacientes dentro de las decisiones sobre el cuidado de salud de los pacientes en forma individual"(17).

Respecto a las etapas para el uso de la evidencia, dos $\operatorname{artículos}^{(6,18)}$ mencionan 4 etapas: 1) Formulación de una pregunta clínica; 2) Búsqueda en la literatura médica; 3) Análisis crítico de la evidencia y 4) Aplicación de los resultados. Otra parte importante de los artículos ${ }^{(1,14,16,18-22)}$ proponen cinco fases y como aplicables en la EBE, se describen a continuación: 1) Elaboración de una pregunta: que nace de la incertidumbre para la toma de decisiones ${ }^{(8,20)}$, las necesidades de información de la práctica se convierten en preguntas enfocadas y estructuradas ${ }^{(20,21)}$ y se recomienda el uso del formato PICO (P: Paciente o problema a estudio, I: Intervención, C: Comparación, O: Resultados (outcomes) ${ }^{(16,19-21)}$; las palabras clave de la pregunta servirán de guía para la siguiente fase; 2) Búsqueda bibliográfica: etapa en que por medio de una estrategia de búsqueda se acerca a la mejor y más actualizada evidencia para resolver la pregunta estructurada; se trabaja en las diferentes bases de datos y como en la actualidad la producción científica avanza rápidamente, se cuenta con revisiones sistemáticas y metaanálisis de los ensayos controlados aleatorizados, que han sido creados para ayudar en esta búsqueda de los mejores resultados ${ }^{(8,}$ 20, 22); 3) Análisis crítico: se valora la calidad de los artículos seleccionados, es decir, si responde a la pregunta estructurada, si le es útil( ${ }^{(8)}$, si cumple desde el punto de vista del rigor metodológico y si los artículos cuentan con la validez interna y externa deseada, aplicando listados de verificación con criterios establecidos ${ }^{(20,22)}$; 4) Implementación o transferencia de la evidencia a la práctica clínica ${ }^{(19}$, ${ }^{20)}$ : una vez seleccionada la evidencia, los resultados de la investigación deben llegar a las instituciones, profesionales sanitarios y pacientes ${ }^{(19,20)}$, implica trabajar con los pares para motivar el cambio en la atención de los cuidados, considerando las preferencias y creencias de los pacientes $^{(22)}$;
5) Evaluación: se trata de medir el efecto de los cambios que se realizaron para la práctica clínica, en relación con los cuidados prestados, se centra en los resultados y en el proceso, puede incluir una auditoria ${ }^{(19)}$ y permite la retroalimentación permanente entre la investigación y la clínica ${ }^{(22)}$.

Barreras y elementos facilitadores para la implementación de la EBE: La evidencia señala que las barreras para la implementación de la EBE se pueden medir a través de la Escala de Barreras, instrumento creado por Funk y colaboradores ${ }^{(23)}$ que consta de 28 ítems y se pueden agrupar en cuatro dimensiones: 1) características del adoptante de la investigación (enfermero), 2) características de la organización (entorno), 3) innovación de la investigación (investigación), y 4) característica de la comunicación de la investigación (presentación).

Tras revisar la literatura, las barreras se pueden agrupar en tres grandes áreas: de los propios profesionales, organizacionales y del entorno (Tabla 3).

Los facilitadores señalados en los artículos son: apoyo del gerente, tiempo y apoyo de las colegas, accesos a resultados, capacitación y educación en investigación, oportunidad para estudios adicionales especialmente en investigación y mejorar la base de investigación de la enfermera en ejercicio ${ }^{(23)}$.

En cuanto a los elementos facilitadores, la literatura revisada muestra que la implicación de los directivos en el proceso es relevante ${ }^{(24-28)}$, así como

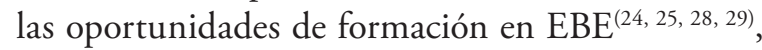
un mayor desarrollo disciplinario (licenciatura, doctorado) ${ }^{(24,30)}$, la creación de equipos de investigación, las organizaciones democráticas y de escucha, la construcción cultural( ${ }^{(31-33)}$, la facilidad en la diseminación y la puesta en práctica de los hallazgos, y el reconocimiento de $\operatorname{los} \operatorname{logros}{ }^{(24)}$. Comprender que la EBE mejora la atención al paciente y la mejora de la credibilidad en enfermería también es mencionado como un facilitador ${ }^{(34)}$. Las funciones de gestión, como la edad más joven ${ }^{(29)}$ también se mencionan como facilitadores y las enfermeras con más años de experiencia informan un mayor uso y actitud positiva hacia la $\mathrm{EBE}^{(35)}$. 
Tabla 3. Barreras para la implementación de EBE.

\begin{tabular}{|c|c|}
\hline \multirow[t]{5}{*}{ De los propios profesionales } & $\begin{array}{l}\text { Falta de conocimiento, conciencia, habilidades en la temática de EBE y evalua- } \\
\text { ción de la evidencia }{ }^{(13,29,30-34,36-43,47)}\end{array}$ \\
\hline & $\begin{array}{l}\text { Prácticas rutinarias, actitudes reacias e inmovilistas, carencia formativa en la } \\
\operatorname{PBE}^{(13,34,36,38,47)}\end{array}$ \\
\hline & La falta del dominio del inglés, aislamiento profesional ${ }^{(24,44,48)}$. \\
\hline & Uso del pensamiento crítico y reflexivo ${ }^{(24)}$ \\
\hline & Falta de tiempo para leer la literatura ${ }^{(44)}$ \\
\hline \multirow[t]{8}{*}{ Organizacionales } & Sobrecarga laboral $(22,25,29,30,35,37,40,41,43-46)$. \\
\hline & Falta de financiamiento, dificultad en el acceso a fuentes $(21,32,33,34,38,39,41,43,45,47,48)$. \\
\hline & Déficit de autonomía de las enfermeras ${ }^{(13,22-25,41,45-47)}$. \\
\hline & $\begin{array}{l}\text { Culturas de gestión obsoletas que no fomentan la innovación en los cuidados } \\
\text { enfermeros } 34,36 \text {. }\end{array}$ \\
\hline & Rotación y movimiento constante del personal ${ }^{(26)}$. \\
\hline & Personal insuficiente ${ }^{(26,38,44)}$. \\
\hline & Pocas o nulas oportunidades para la educación y capacitación en $\mathrm{EBE}^{(26,38,41,47)}$. \\
\hline & $\begin{array}{l}\text { Falta de compromiso organizacional con el programa de EBE, especialmente a } \\
\text { nivel ejecutivo }{ }^{(13,26,27,31,37,38,47)} \text {. }\end{array}$ \\
\hline \multirow[t]{3}{*}{ Del entorno } & Conducta de oposición de otros profesionales, pacientes y familiares ${ }^{(36)}$ \\
\hline & Falta de apoyo y liderazgo de los directivos de las instituciones ${ }^{(27,38)}$ \\
\hline & Falta de comunicación entre los entornos de práctica académica y clínica ${ }^{(27,40)}$ \\
\hline
\end{tabular}

\section{DISCUSIÓN}

Con esta revisión de alcance se han podido recoger los orígenes de la investigación para la toma de decisiones en Medicina hasta lo que actualmente se conoce como Epidemiología Clínica, base de la MBE. Archibald Cochrane destaca como uno de los precursores de este movimiento ${ }^{(1,6-14)}$ y si bien es Cochrane el que desencadena y motiva al estamento médico en el uso de la investigación para la toma de decisiones, Gordon Guyatt es quién finalmente acuñó el término de $\mathrm{MBE}^{(1,7,8)}$. Desde entonces a la fecha se ha recomendado el uso de esta herramienta en el estamento médico considerando las preferencias y creencias de los pacientes para la toma de decisiones sobre la práctica clínica.

Importante es que se mencione a Florence Nightingale como una estadística que tuvo gran relevancia por el uso de la evidencia en salud, a pesar de que en esa época aún no existía el concepto $(7,10,15)$, en su libro "Notas sobre Enfermería ${ }^{(49)}$ ¿Qué es y qué no es?", sus escritos reflejan el interés por la investigación en aquellos factores ambientes que influían en el bienestar físico y emocional de las personas, menciona la ventilación, la limpieza, la pureza del agua y la alimentación. Otro aspecto interesante es el valor que le brindaba a la observación para crear pruebas sólidas que influyeron en la salud y los resultados de los pacientes. Para el colectivo de enfermería el uso de la investigación debe entenderse como una estrategia que apoya la práctica aumentando los beneficios para la disciplina al aportar nuevos conocimientos y a las personas al brindarles cuidados de calidad.

Los pasos que ha seguido la evolución de la EBE son coincidentes en los artículos revisados ${ }^{(13,14,17)}$, en los que se estable sus inicios en países como Canadá, Estados Unidos y Reino Unido. Cabe destacar que sólo uno de estos artículos ${ }^{(17)}$ atribuye los primeros indicios a un estudio de cuidados de enfermería del Royal College of Nursing del Reino Unido, a diferencia de los artículos encontrados 
sobre los orígenes de MBE, lo que de alguna manera podría reflejar la escasa visibilidad que ha tenido este proceso en enfermería.

En definitiva, y como resultado de la revisión del término "Historia" (de MBE y EBE); y del concepto y la función de la EBE, señalamos que todas las definiciones concuerdan en la importancia del uso de la evidencia científica en la toma de decisiones para lograr un cuidado de calidad. Algunas destacan, además la necesidad de que el profesional efectúe un análisis crítico y reflexivo. Sin embargo, si bien todas las definiciones están orientadas a beneficiar a los pacientes con el uso de la investigación, no todas incluyen las preferencias de estos en el momento de la toma de decisiones.

Como se ha visto, estas definiciones nacen de la Medicina Basada en la Evidencia desde su mirada más positivista, pero se ha ido adaptando hacia Enfermería, tal como lo refleja la definición elaborada en la Primera Reunión de EBE en 2002 en Granada, España, orientada bastante más al paradigma cualitativo.

La definición más cercana a una mirada integral de cuidado basado en la evidencia, sería una desde los dos paradigmas investigativos, incorporados al conocimiento enfermero y sus cuidados, considerando también lo mencionado por Ingersoll, es decir, lo relevante de hacer un uso juicioso de la investigación científica nos debe hacer reflexionar, para no hacer de ella un uso sin conciencia e incluir al paciente en la toma de decisiones, como protagonista de su cuidado.

Por el análisis crítico del concepto de EBE, se pudo observar que las palabras que más se repiten en las definiciones citadas son: uso consciente, explícito y juicioso ${ }^{(1,9,13)}$, toma de decisiones ${ }^{(1,7,9)}$ y preferencia del paciente ${ }^{(1,7,9,16)}$. La presencia de este último posiblemente se deba a la integración del paradigma cualitativo en el desarrollo de la EBE, que la ha acercado más hacia el eje disciplinar de Enfermería, el cuidado, el cual surge a partir de las necesidades del paciente.

Con todo, la EBE es una herramienta que permite a los profesionales de enfermería aplicar la mejor evidencia disponible para la resolución de problemas en la práctica clínica, en la gestión o en la academia y su sistematización en cinco etapas o pasos ${ }^{(1,14,16,19-22)}$ facilitan su aplicación, al proporcionar una guía útil, incluso para enfermeras noveles. La enseñanza de esta herramienta a los futuros profesionales requiere una apropiación de la metodología por parte de los docentes, pues muchos de ellos sólo se han formado bajo el concepto de la investigación para el levantamiento de nuevo conocimiento, pero la EBE es una estrategia para el uso de la investigación existente en la resolución de problemas y toma de decisiones para el cuidado. Dado lo anterior, es recomendable que tanto en las instituciones de salud como en la academia existan tutores que acompañen en la formación y uso de la EBE, intencionando el aprendizaje de los tres primeros pasos del proceso de la EBE que aportan los elementos básicos para el dominio de esta herramienta.

En relación con las barreras para el uso de la EBE, se constata que muchas de ellas han permanecido en el tiempo, a pesar de los años que han pasado desde el inicio de este movimiento. Las que más se repiten surgen del propio profesional de enfermería ${ }^{(13,23,29-32,34,36-42)}$. Entre ellas, la falta del dominio del idioma inglés, que reduce la referencia y lectura de artículos y se mantiene el llamado aislamiento profesional ${ }^{(24)}$, relevante en América Latina o en los países de Asia, en donde se afirma que existe una escasez de replicación de evidencia en chino, lo que ha significado una barrera para la implementación de la Enfermería Basada en la Evidencia en ese continente ${ }^{(24,39,40)}$.

Las barreras que se originan de la organización de su trabajo ${ }^{(22,25,29,30,34,35,40,41,43-46)}$, de la falta de compromiso organizacional por parte de los ejecutivos ${ }^{(13,26-28,31,37-39)}$ y de la lentitud en publicar la evidencia, según la visión de los estudiantes ${ }^{(46)}$, han sido importantes, difíciles de eliminar y que permiten justificar el escaso desarrollo de esta práctica por parte de los profesionales de enfermería. Si bien los artículos revisados en su mayoría son investigaciones desde el paradigma cuantitativo, los estudios cualitativos revisados también identifican obstáculos para la incorporación de la evidencia, relacionados con los propios profesionales, con el contexto social y con el contexto organizacional ${ }^{(36)}$.

A la vista de estos resultados es pertinente señalar que las barreras para la implementación de EBE deben ser abordadas también desde la formación de los futuros profesionales. Se vuelve a relevar la importancia de contar con académicos preparados para este desafío en la formación, aun cuando también presenten barreras para su enseñanza ${ }^{(33)}$.

Respecto a los facilitadores señalados ${ }^{(33)}$, no se 
debe olvidar la importancia de realizar el cuidado a la cabecera del paciente y cultivar un cuidado enmarcado en todos los aspectos de la humanización que lo sustenta, ya que se trata del punto de partida para generar preguntas de investigación y aumentar el cuerpo de conocimientos de la disciplina. La formación permanente y actualizada a los profesionales de enfermería en EBE es la herramienta principal para lograr este cambio que brinda cuidados seguros y de calidad a la población, resultando clave el papel de las universidades para el logro de estas metas.

Una de las limitaciones de esta revisión es que representa parte de la realidad del mundo occidental, si bien se rescataron artículos de otras culturas, esto fue posible a que se encontraban en inglés, pero fueron escasos. Este trabajo es una primera revisión y se optó por bases de datos originarias, es decir, anglosajonas. Se logra rescatar algunos artículos de países de Asia que muestran sus realidades, pero dada las limitaciones en el idioma no permite conocer el desarrollo de esta herramienta en mayor profundidad a nivel global. Sería conveniente considerar estos elementos para próximas revisiones del tema como también incluir más bases de datos latinoamericanas o europeas que cuentan con una amplia cobertura de producción científica de Enfermería.

\section{CONCLUSIONES}

La EBE es una estrategia en el uso de la evidencia científica para la toma de decisiones frente a los cuidados que aportan los profesionales de Enfermería. A Florence Nightingale se le reconoce como la primera enfermera que apoyó su trabajo con pruebas sólidas.

EBE nace de la MBE, su desarrollo como tal se describe a partir de 1997 contribuyendo a los cuidados seguros y de calidad para las personas, beneficiando a las instituciones de salud al disminuir costos y entregando seguridad, empoderamiento y liderazgo en los cuidados a la disciplina de Enfermería.

Las principales barreras encontradas para la implementación de la EBE se relacionan con los propios profesionales de enfermería, su entorno y de las propias organizaciones, muchas de las cuales, a pesar de los años de desarrollo, aún persisten.
Es necesario considerar las barreras y facilitadores para diseñar estrategias eficaces para su implementación, tanto en el ámbito asistencial, como docente y gestor.

\section{REFERENCIAS}

1. Beyea C S, Slattery MJ. Historical Perspectives on Evidence-Based Nursing. Nurs Sci Q [Internet]. 2013 [citado 09 abr 2019]; 26(2):152-5. Disponible en: https://doi.org/10.1177\%2F089431 8413477140

2. Peters J, Godfrey C, McInerney P, Munn Z, Tricco C, Khalil, H. In: Aromataris E, Munn Z (Editors). JBI Manual for Evidence Synthesis [Internet]. JBI; 2020 [citado 05 ene 2021]. Disponible en: https:// doi.org/10.46658/JBIMES-20-12

3. Peters J, Godfrey C, McInerney P, Khalil, $\mathrm{H}$. Deborah PS, Cassia B. Guidance for conducting systematic scoping reviews. International Journal of Evidence-Based Healthcare [Internet] 2015 [citado 08 dic 2021]; 13(3): 141-146. Disponible en: https://doi.org/10.1097/xeb.0000000000000050

4. Pearcey A. Achieving Research-Based Nursing Practice. JAN [Internet]. 1995 [citado 13 ago 2021]; 22(1): 33-39. Disponible en: https://doi. org/10.1046/j.1365-2648.1995.22010033.x

5. CASPE. Programa de Habilidades en Lectura Crítica Español [Internet]. España [citado 25 ago 2021]. Disponible en: https://www.redcaspe.org/ herramientas/instrumentos

6. Martí CA. La medicina basada en evidencia en 1948: visión desde 2005. Gac Méd Caracas [Internet] 2006 [citado 10 dic 2020]; 114(3): 208213. Disponible en: http://ve.scielo.org/scielo. php?script=sci_arttext\&pid=S036747622006000 300006\&lng=es.

7. Mackey A, Bassendowski S. The History of Evidence-Based Practice in Nursing Education and Practice. J Prof Nurs [Internet]. 2017 [citado 09 abr 2019]; 33(1): 51-55. Disponible en: https:// doi.org/10.1016/j.profnurs.2016.05.009

8. Sánchez LK, Sosa SR, Green RD, Motola KD. Importancia de la medicina basada en evidencias en la práctica clínica cotidiana. Médica Sur [Internet]. 2007 [citado 05 may 2020]; 14(1): 9-13. Disponible en: https://www.medigraphic. com/pdfs/medsur/ms-2007/ms071b.pdf.

9. Scott K, McSherry R. Evidence-based nursing: clarifying the concepts for nurses in practice. J Clin Nurs [Internet]. 2009 [citado 09 abr 2019]; 18(8): 1085-95. Disponible en: https://doi. org/10.1111/j.1365-2702.2008.02588.x 
10. Brower EJ, Nemec R. Origins of EvidenceBased Practice and what it Means for Nurses. Int J Childbirth Educ [Internet]. 2017 [citado 05 may 2020]; 32(2): 14-18. Disponible en: https:// www.coursehero.com/file/39840949/Origins-ofEvidence-Based-Practice-and-What-it-Means-forNursespdf/

11. Mazurek B, Fineout-Overholt E, Stone P, Ackerman M. Evidence-based practice: the past, the present and recommendations for the millennium. Pediatric Nursing [Internet]. 2000 [citado 05 may 2020]; 26(1): 77-80. Disponible en: https://pubmed.ncbi.nlm.nih.gov/12026318/

12. Roy LS. Evidence-based nursing offers certainty in the uncertain world of healthcare. Nursing Management [Internet]. 2004 [citado 07 jun 2020]; 35(10): 10-12. Disponible en: https:// pubmed.ncbi.nlm.nih.gov/15638444/

13. Alcolea M, Oter C, García M. Enfermería Basada en la Evidencia. Orígenes y fundamentos para una práctica enfermera basada en la evidencia. Nure Investigación [Internet]. 2011 [citado 07 jun 2020]; 52: 1-7. Disponible en: http://www. nureinvestigacion.es/OJS/index.php/nure/article/ download/538/527/

14. Noe E. Enfermería Basada en la Evidencia, una ruta hacia la aplicación en la práctica profesional. Enfermería en Costa Rica [Internet]. 2011 [citado 10 jun 2020]; 32(1): 89-96 Disponible en: https:// www.researchgate.net/publication/305681200_ RESUMEN_ABSTRACT_Evidence-Based_ Nursing_A_Route_To_Its_Aplication_Into_ Practice

15. McDonald L. Florence Nightingale and the early origins of evidence-based nursing. Evid Based Nurs [Internet]. 2001 [citado 20 jul 2020]; 4(7): 68-69. Disponible en: https://ebn.bmj.com/ content/ebnurs/4/3/68.full.pdf

16. Estabrooks CA. Thoughts on Evidence-based nursing and its science-A Canadian perspective. Worldviews Evid-Based Nurs. [Internet]. 2004 [citado 20 jul 2020]; 1(2): 88-91. Disponible en: http://dx.doi.org/10.1111/j.17416787.2004.04037.x

17. Urra ME, Retamal VC, Tapia PC, Rodríguez VM. Enfermería Basada en la Evidencia: qué es, sus características y dilemas. Invest Educ Enferm [Internet]. 2010 [citado 20 may 2020]; 28(1): 10818. Disponible en: http://www.scielo.org.co/pdf/ iee/v28n1/v28n1a13.pdf

18. Letelier SL, Moore P. La medicina basada en evidencia. Visión después de una década. Rev Méd Chile [Internet]. 2003 [citado 20 jun 2020]; 131(8): 939-946. Disponible en: http://dx.doi. org/10.4067/S0034-98872003000800016
19. Alcolea M, Oter C, García M. Fases de la Práctica Basada en la Evidencia. Nure Investig [Internet]. 2011 [citado 14 ago 2019]; 8(53): 1-5. Disponible en: https://www.nureinvestigacion.es/OJS/index. $\mathrm{php} /$ nure/article/view/545/534

20. Icart IM. La Evidencia Científica: Estrategia para la práctica enfermera. ROL Enf. [Internet]. 1999 [citado 20 ago 2019]; 22(3): 185-190. Disponible en: http://hdl.handle.net/2445/102730

21. Flemming K. Asking answerable questionsEvidence-Based Nursing. Evid Based Nurs [Internet]. 2001 [citado 20 ago 2019]; 1(2): 6869. Disponible en: https://ebn.bmj.com/content/ ebnurs/1/2/36.full.pdf

22. Orellana YA, Paravic KT. Enfermería Basada en la Evidencia. Barreras y estrategias de implementación. Cienc enferm [Internet]. 2007 [citado 10 feb 2020]; 13(1): 17-24. Disponible en: https://scielo.conicyt.cl/pdf/cienf/v13n1/art03. pdf.

23. Parahoo K. Barriers to, and facilitators of, research utilization among nurses in Northern Ireland. J Adv Nurs [Internet]. 2000 [citado 12 feb 2019]; 31(1): 89-98. Disponible en: https://doi. org/10.1046/j.1365-2648.2000.01256.x

24. Martínez J. Barreras e instrumentos facilitadores de la enfermería basada en la evidencia. Enferm Clín [Internet]. 2003 [citado 18 oct 2020]; 13(5): 30308. Disponible en: https://pdf.sciencedirectassets. com/277721/1-s2.0-S1130862103X7018871

25. Gea-Sánchez M. Conocimiento, actitudes y barreras percibidas por las enfermeras para integrar evidencia científica en la práctica clínica en un hospital universitario. Enferm Clín [Internet]. 2010 [citado 13 dic 2020]; 20(5): 313-14. Disponible en: doi.org/10.1016/j.enfcli.2010.07.002

26. Sharplin G, Adelson P, Kennedy K, Williams N, Hewlett R, Wood J, et al. Establishing and Sustaining a Culture of Evidence-Based Practice: An Evaluation of Barriers and Facilitators to Implementing the Best Practice Spotlight Organization Program in the Australian Healthcare Context. Healthcare (Basel) [Internet]. 2019 [citado 10 oct 2020]; 7(4): E142. Disponible en: https://doi.org/10.3390/healthcare7040142

27. Melnyk B, Fineout-Overholt E, Gallagher-Ford L, Kaplan L. El estado de la práctica basada en la evidencia en las enfermeras de Estados Unidos: implicaciones críticas para las enfermeras dirigentes y educadores. J Nurs Adm [Internet]. 2012 [citado 10 dic 2020]; 42(9): 410-7. Disponible en: https://pubmed.ncbi.nlm.nih.gov/22922750/

28. Mathieson A, Grande G, Luker K. Strategies, facilitators and barriers to implementation of evidence-based practice in community nursing: 
a systematic mixed-studies review and qualitative synthesis. Prim Health Care Res Dev [Internet]. 2019 [citado 10 ene 2021]; 20: e6. Disponible en: https://dx.doi.org/10.1017\%2FS14634236180 00488

29. Li S, Cao M, Zhu X. Evidence-based practice: Knowledge, attitudes, implementation, facilitators, and barriers among community nurses-systematic review. Medicine (Baltimore) [Internet]. 2019 [citado 20 may 2020]; 98(39): e17209. Disponible en: https://pubmed.ncbi.nlm.nih.gov/31574830/

30. Koehn L, Lehman K. Nurses' perceptions of evidence-based nursing practice. J Adv Nurs [Internet]. 2008 [ citado 15 ago 2020]; 62(2): 20915. Disponible en: https://pubmed.ncbi.nlm.nih. gov/18394033/

31. Yong Yoo J, Hee Kim J, Sun Kim J, Lye Kim H, Suk Ki J. Clinical nurses' beliefs, knowledge, organizational readiness and level of implementation of evidence-based practice: The first step to creating an evidence-based practice culture. PLoS One [Internet]. 2019 [citado 03 abr 2020]; 14(12): e0226742. Disponible en: https://dx.doi. org/10.1371\%2Fjournal.pone.0226742

32. Alqahtani N, Kyeung M, Kitsantas P. Nurses' evidence-based practice knowledge, attitudes and implementation: A cross-sectional study. J Clin Nurs [Internet] 2019 [citado 15 abr 2020]; 29(12):274-283. doi: 10.1111/jocn.15097. Epub 2019 Nov 26. Disponible en: https://onlinelibrary.wiley. com/doi/abs/10.1111/jocn.15097

33. Oh E, Yang Y, Yoo J, Lim J, Sung J. Mixed Method Research Investigating Evidence-Based Practice Self-efficacy, Course Needs, Barriers, and Facilitators: From the Academic Faculty and Clinical Nurse Preceptors. J Korean Acad Nurs [Internet]. 2016 [citado 15 oct 2020]; 46(4):50113. Disponible en: 10.4040/jkan.2016.46.4.501.

34. Camargo F, Iwamoto $\mathrm{H}$, Galvão $\mathrm{C}$, Pereira $\mathrm{A}$, Andrade B, Masso C. Competences and Barriers for the Evidence-Based Practice in Nursing: an integrative review. Rev Bras Enferm [Internet]. 2018 [citado 25 sep 2020]; 71(4): 2030-2038. Disponible en: https://pubmed.ncbi.nlm.nih.gov/ 30156693/

35. Ali A, Ammouri A, Raddaha P, Renu J, Noronha A, Obeidat L. Evidence-Based Practice: Knowledge, attitudes, practice and perceived barriers among nurses in Oman. Sultan Qaboos Univ Med J [Internet]. 2014 [citado 18 feb 2021]; 14(4); e53745. Disponible en: https://pubmed.ncbi.nlm.nih. gov/25364558/

36. Sánchez-García I, López-Medina I, PancorboHidalgo P. Obstáculos percibidos por las enfermeras para la práctica basada en evidencias:
Un estudio cualitativo. Enferm Clín [Internet]. 2013 [citado 20 mar 2020]; 23(6): 279-83. Disponible en: 10.1016/j.enfcli.2013.09.001

37. Hasanpoor ES, Janati A, Hajebrahimi S, Haghgoshayie E. Nursing Managers' Perspectives on the Facilitators and Barriers to Implementation of Evidence-Based Management. Worldviews Evid Based Nurs [Internet]. 2019 [citado 14 sep 2020]; 16(4): 255-262. Disponible en: 10.1111/ wvn.12372.

38. Gifford W, Zhang Q, Chen S, Davies B, Xie R, Wen S-W, et al. When east meets west: a qualitative study of barriers and facilitators to evidence-based practice in Hunan China. BMC Nurs [Internet]. 2018 [citado 01 oct 2020]; 26: 17:1 Disponible en: 10.1186/s12912-018-0295-x.

39. Duncombe D. A multi-institutional study of the perceived barriers and facilitators to implementing evidence-based practice. J Clin Nurs [Internet]. 2018 [citado 10 oct 2020]; 27(5-6): 1216-1226. Disponible en: 10.1111/jocn.14168.

40. Ubbink D, Vermeulen A, Knops D, Legemate K, Oude M, Heineman Y, et al. Implementation of evidence-based practice: outside the box, throughout the hospital. Neth J Med [Internet]. 2011 [citado 25 oct 2020]; 69(2): 87-94. Disponible en: https://pubmed.ncbi.nlm.nih.gov/21411849/

41. Hajbaghery A. Factors facilitating and inhibiting evidence-based nursing in Iran. J Adv Nurs [Internet]. 2007 [citado 20 ago 2020]; 58(6): 56675. Disponible en: https://pubmed.ncbi.nlm.nih. gov/17442028/

42. Thompson C, McCaughan D, Cullum N, Sheldon T, Raynor P. Barriers to evidence-based practice in primary care nursing-why viewing decisionmaking as context is helpful. J Adv Nurs [Internet]. 2005 [citado 04 nov 2020]; 52(4):432-44. Disponible en: 10.1111/j.1365-2648.2005.03609.x

43. Majid S, Foo S, Luyt B, Zhang X, Theng Y, Chang Y, Mokhtar I. Adopting evidence-based practice in clinical decision making: nurses' perceptions, knowledge, and barriers. J Med Libr Assoc. [Internet] 2011 [citado 20 oct 2020]; 99(3):22936. Disponible en: 10.3163/1536-5050.99.3.010.

44. Khammarnia M, Mohammadi M, Amani Z, Rezaeian S, Setoodehzadeh F. Barriers to Implementation of Evidence Based Practice in Zahedan Teaching Hospitals, Iran, 2014. Nurs Res Pract [Internet]. 2015 [citado 08 oct 2020]; 2015: 357140. Disponible en: 10.1155/2015/357140.

45. Labrague L, McEnroe-Petitte D, D'Souza C, Fronda D, Edet $\mathrm{O}$, Ibebuike J, et al. A Multicountry Study on Nursing Students' SelfPerceived Competence and Barriers to EvidenceBased Practice. Worldviews Evid Based Nurs 
[Internet]. 2019 [citado 06 feb 2021]; 16(3): 236246. Disponible en: 10.1111/wvn.12364.

46. Brown EC, Wickline MA, Ecoff L, Glaser D. Nursing practice, knowledge, attitudes and perceived barriers to evidence-based practice at an academic medical center. J Adv Nurs [Internet]. 2009 [citado 08 ene 2021]; 65(2):371-81. Disponible en: 10.1111/j.1365-2648.2008.04878.x.

47. DeBruyn R, Ochoa-Marín S, Semenic S. Barriers and facilitators to evidence based nursing in Colombia: perspectives of nurse educators, nurse researchers and graduate students. Invest Educ Enferm [Internet]. 2014 [citado 09 nov 2020];
32(1): 9-21. Disponible en: doi: 10.17533/udea.iee. v32n1a02.

48. Sun-Ae Kim, Youngshin S, Hee-Sook S, EunKyong A, Jung-Hee K. Mediating role of critical thinking disposition in the relationship between perceived barriers to research use and evidencebased practice. Contemp Nurse [Internet]. 2015 [citado 25 oct 2020]; 51(1): 16-26. Disponible en: 10.1080/10376178.2015.1095053.

49. Nightingale F. Notas sobre Enfermería. ¿Qué es qué no es? Editorial Elsevier Masson. $1^{\text {ra }}$ edición; 1990. 